\title{
Vehicle License Plate Detection and Recognition System
}

\author{
Poonam Bhogale \\ K J Somaiya College of Engineering
}

\author{
Archit Save \\ $\mathrm{K} J$ Somaiya College of \\ Engineering
}

\author{
Vitrag Jain \\ K J Somaiya College of \\ Engineering
}

\author{
Saurabh Parekh \\ $\mathrm{K} J$ Somaiya College of \\ Engineering
}

\begin{abstract}
This is a survey paper on the Vehicle License Plate Detection and Recognition System. This system works in five phases. The first phase is the input given to the system which is an image of the vehicle with clearly visible license plate. The second phase is the localization of the license plate from the image. The third phase is the segmentation of the characters in the localized license plate. In the fourth phase the segmented characters are recognized using Artificial Neural Networks. In the fifth and final phase these recognized characters are stored in a database for further reference.
\end{abstract}

\section{General Terms}

Automatic License Plate Recognition (ALPR), MATLAB, Neural Networks

\section{Keywords}

Artificial Neural Networks, database, histogram, region splitting, Vehicle License Plate Detection and Recognition System

\section{INTRODUCTION}

The world is moving at a very fast pace. With new technologies coming up, the human effort to get things done is decreasing and life is becoming easier. One of the major aspects of this fast moving life is the digitalization of everyday articles. For digitization the images taken from a camera play a very important role as these images can be processed using various techniques and algorithms to extract useful information from them. For instance extracting text from an image can be very difficult but can be achieved using various character recognition techniques and algorithms.

This paper surveys the technique and method to store the number on the license plate from the image of a vehicle. This paper presents the proceedings to be carried out by the system after taking the image of the vehicle and how they can be done. Firstly, the image of the vehicle is given as input to the system. By analyzing the histograms of the image, the license plate is detected in the image and is localized by isolating the license plate. Using region splitting, the characters in the localized license plate are segmented. These segmented characters are recognized using Artificial Neural Networks which have been trained using a feed-forward network by supervised learning. These recognized characters are then stored in a database which can be used for reference later (Refer Fig. 1). This system can be implemented by the police to identify stolen vehicles, collecting toll tax at a toll booth or at unmanned parking lots.

This paper will now present the phases through which the system goes to carry out the proceedings in detail.

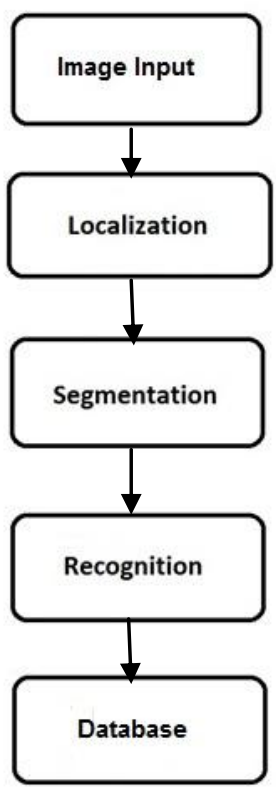

Fig. 1

\section{IMAGE INPUT}

The image that is provided as input to the system has to satisfy certain conditions (Refer Fig. 2)

- The license plate of the vehicle should be clearly visible in the image

- The image itself should be clear enough for the system to recognize.

- Preferably, the images of the vehicle should be straight of the front-end or straight of the back-end

There can be some exceptional cases where the system may not function properly and may give inaccurate results such as if the license plate is damaged or is rusted. Also, if the font used in the license plate is different and not a standard one. 


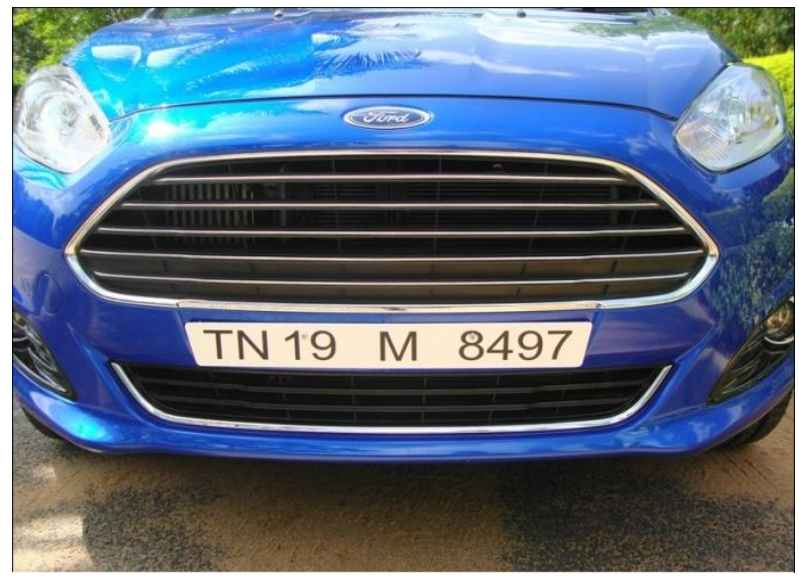

Fig. 2

\section{LOCALIZATION}

Localization takes place using histograms. Once the image is given as input, the image is converted to a gray scaled image (Refer Fig. 3). Dilation and Erosion operations are performed on it to remove the noise from the image. After that, the edges are processed horizontally to plot the horizontal histogram for analysis. The horizontal histogram needs to be smoothened; hence, a low pass filter is applied to it. Later on, the horizontal histogram values are filtered out by applying a dynamic threshold (Refer Fig. 4). The same procedure is repeated for edges vertically and in this case a vertical histogram is plot (Refer Fig. 5). Once the system analyzes the horizontal and vertical histograms, the probable candidates for a license plate in the image are identified (Refer Fig. 6). These candidates are the region of interest which are extracted. On further processing, the most probable region of interest is identified which is isolated and thus, the localization of the license plate takes place (Refer Fig. 7). This localized license plate is then passed on to the next phase for segmentation. The images presented for localization are a result of the partial implementation of the system.

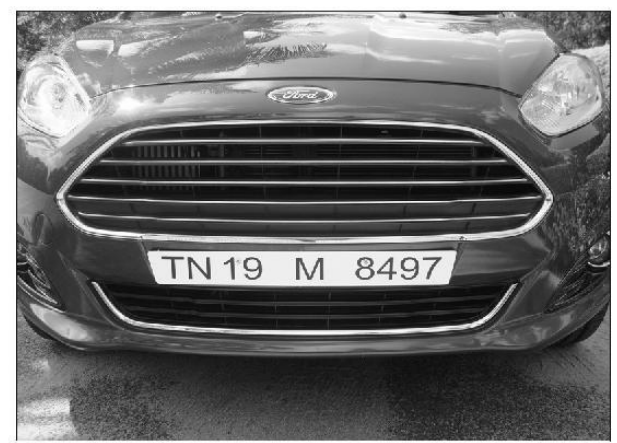

Fig. 3
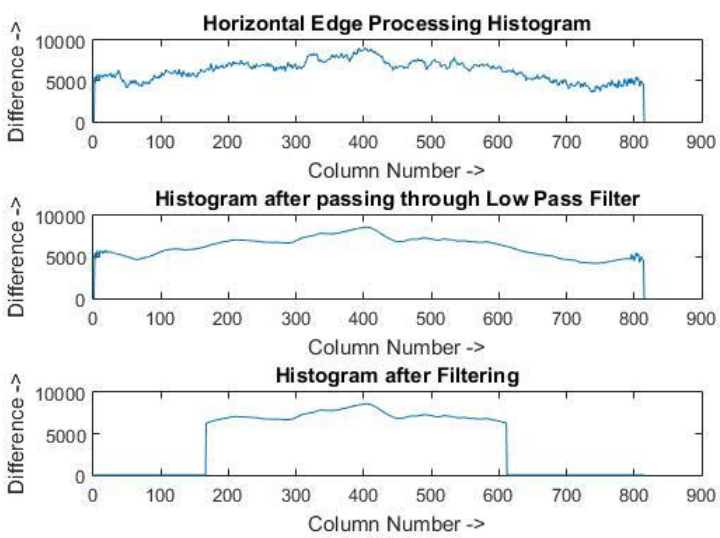

Fig. 4
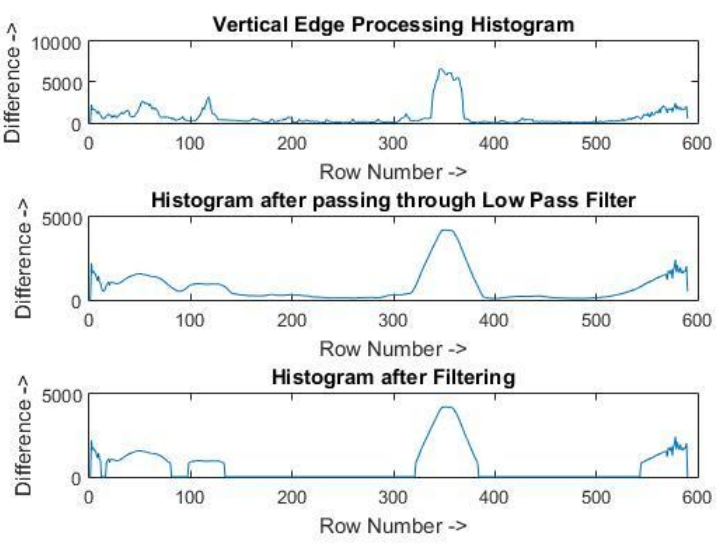

Fig. 5

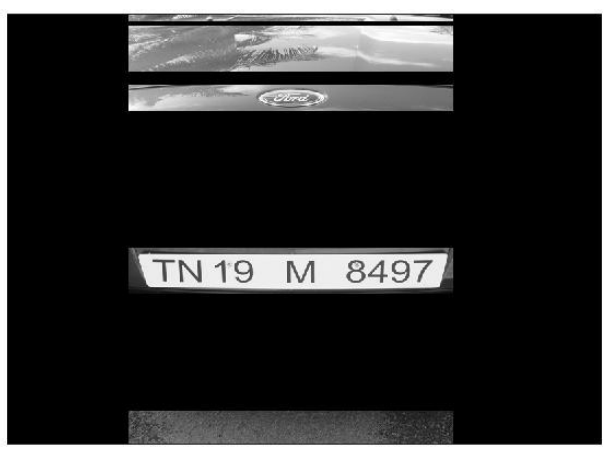

Fig. 6 


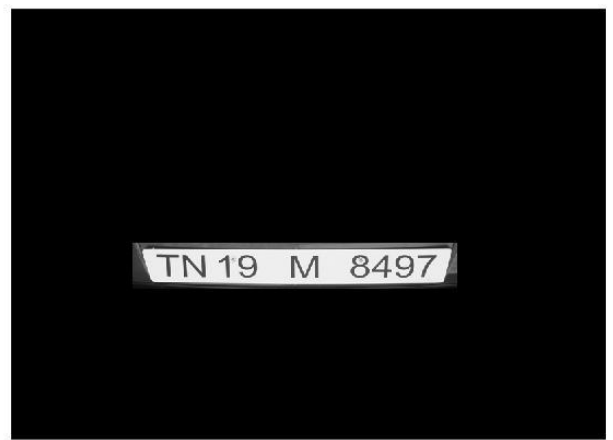

Fig. 7

\section{SEGMENTATION}

The localized license plate goes as input for segmentation of the characters. The gray scaled image is converted to a binary image and all the objects containing less than 30 pixels are removed. This removes the noise from the image. The connected components are labeled and the image region properties are measured in this noise free image and thus, a bounding box is plot (Refer Fig. 8). Finally, the objects i.e. the characters are extracted individually which will be further
$T$

N

1
9
M

$$
7
$$

passed on to the Artificial Neural Networks for recognition (Refer Fig. 9). The images presented for segmentation are a result of the partial implementation of the system.

INPUT IMAGE WITHOUT NOISE

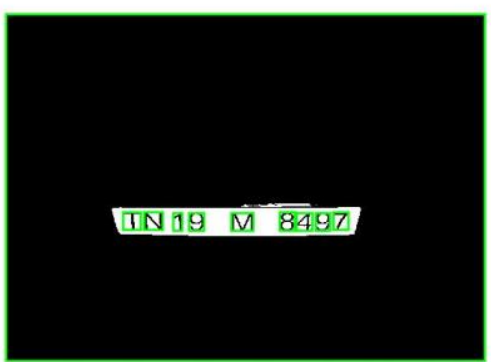

Fig. 8
Fig. 9

\section{RECOGNITION}

The recognition part is implemented using Artificial Neural Networks available in the Neural Network toolkit in MATLAB. To use Neural Networks, they first need to be trained; for training for character recognition supervised learning technique is used. In this type of training, an input is given which has a corresponding output with it which is the desired output from the network. Feature extraction is used to extract the peculiar features using which the characters can be distinguished. A comparison between these features of the actual output and the desired output computes the error which is used to adjust the parameters of the Neural Network like connection weights and thresholds. This modification of the parameters improves the performance of the Neural Networks and is can be continued till desired output is achieved.

\subsection{Types of Neural Network}

There are two types of Neural Networks that can be used to implement character recognition.

- $\quad$ Single Layer Feed-forward Network

- Multilayer Feed-forward Network

\subsubsection{Single Layer Feed-forward Network [6]}

Single layer feed-forward network consists of perceptrons in only two layers; the input layer and the output layer which are connected to each other by weights. This type of network does not have a feedback and hence, the present output does not influence the future outputs (Refer Fig. 10).

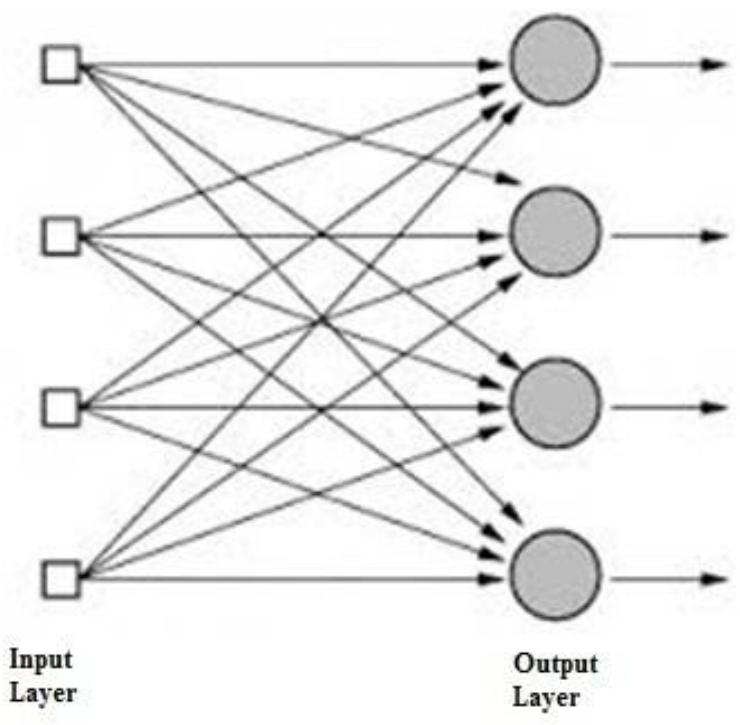

Fig. 10

Perceptron Learning Rule:

$$
\begin{aligned}
& \text { net }_{\mathrm{i}}=\mathrm{W}_{\mathrm{i}} * \mathrm{X} \\
& \mathrm{O}_{\mathrm{i}}=\operatorname{sign}\left(\text { net }_{\mathrm{i}}\right) \\
& \alpha \mathrm{W}=\mathrm{c}\left(\mathrm{d}-\mathrm{O}_{\mathrm{i}}\right) \mathrm{X}
\end{aligned}
$$


where,

$\mathrm{W}_{\mathrm{i}}$ : Weight Vector

$\mathrm{X}$ : Input Vector

O: Obtained Output

d: Desired Output

$\alpha \mathrm{W}$ : Weight Error

The Weight Vector updates using the formula:

$$
\mathrm{W}_{\mathrm{i}+1}=\mathrm{W}_{\mathrm{i}}+\alpha \mathrm{W}
$$

Thus, by updating the Weight Vector desired output can be obtain the.

\subsubsection{Multilayer Feed-forward Network [7]}

Multilayer feed-forward network consists of perceptrons in three or more layers; the input layer, the output layer and at least one hidden layer which connects the input and output layers. This type of network uses back-propagation for adjusting the parameters of the Neural Network (Refer Fig. $11)$.

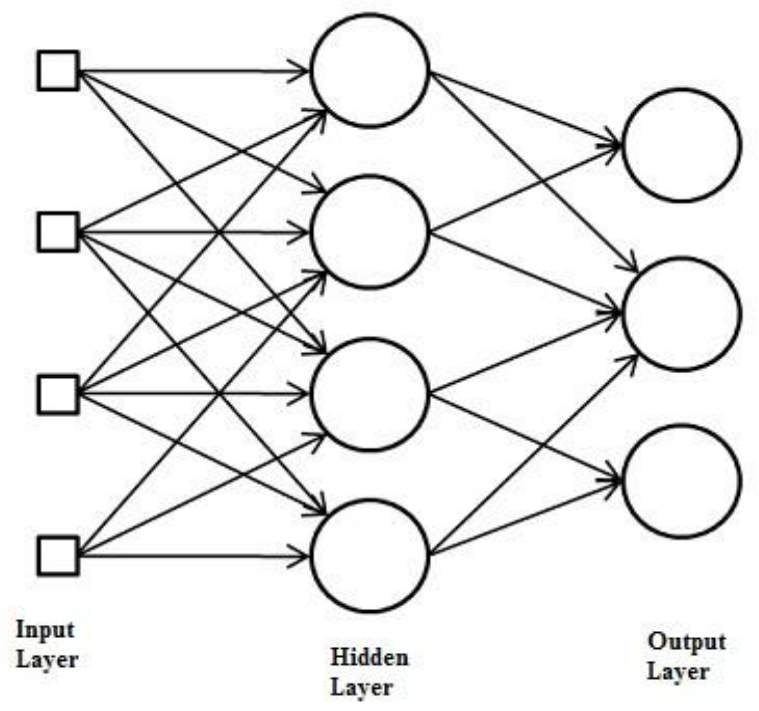

Fig. 11

Equation for Multilayer Feed-forward Network:

$\mathrm{y}_{\mathrm{k}}(\mathrm{t}+1)=\mathrm{F}_{\mathrm{k}}\left(\mathrm{s}_{\mathrm{k}}(\mathrm{t})\right)=\mathrm{F}_{\mathrm{k}}\left(\sum \mathrm{w}_{\mathrm{jk}}(\mathrm{t}) \mathrm{y}_{\mathrm{j}}(\mathrm{t})+\Theta_{\mathrm{k}}(\mathrm{t})\right)$

\section{DATABASE}

After the character recognition process, each and every character is stored in a dataset. The dataset consist of the license plate number, the date and the time when the system recognized the license plate. This dataset is then exported to MySQL database created in MATLAB itself using Database toolkit. This data can be retrieved and processed from the database using simple MySQL queries.

\section{TEST CASES}

For test cases various images have been considered. These images have many variations in terms of font size, font style, font color and the angle from which they were taken. These test cases also have variations in backgrounds like during the day and during the night.100 different images have been considered of which 53 were properly localized and segmented. The recognition part is yet to be implemented for which work is in progress.

\section{CONCLUSION}

MATLAB has all the functionalities required to implement the system. Instead of using different software tools and figuring out ways to merge and implement, MATLAB provides all the toolboxes on a single platform for easy execution of the system. The use of histograms in the localization phase makes it easy to understand what pixel values are required to locate the license plate in the image. For the recognition process, the use of connected components helps us to differentiate and separate out each and every character from the extracted license plate. These characters are then recognized using Neural Networks via the neural network toolbox. The main reason to use Artificial Neural Networks is because of it learning nature, by training the Neural Networks; it can recognize various styles of alphabets and numbers. Even if the Neural Network may give inaccurate results, it keeps on learning and can give better results.

\section{REFERENCES}

[1] Eva Volna, Martin Kotyrba, "Vision System for License Plate Recognition based on Neural Networks", IEEE, 2013.

[2] Fajas F, Farhan Yousuf, Remya P.R. "Automatic Number Plate Recognition for Indian Standard Number Plates", IEEE First Workshop on Emerging Technologies and Devices in Signal Processing, 2012.

[3] Hanit Karwal, Akshay Girdhar, "Vehicle Number Plate Detection System for Indian Vehicles", IEEE International Conference on Computational Intelligence \& Communication Technology, 2015.

[4] Jitendra Sharma and Amit Mishra and Khushboo Saxena "A Hybrid Technique for License Plate Recognition Based on Feature Selection of Wavelet Transform and Artificial Neural Network", IEEE International Conference on Reliability, Optimization and Information Technology, 2014.

[5] Priyanka Prabhakar, Anupama P, Resmi S R, "Automatic Vehicle Number Plate Detection and Recognition”, IEEE International Conference on Control, Instrumentation, Communication and Computational Technologies, 2014.

[6] Dinesh Dileep, "A Feature Extraction Technique Based on Character Geometry for Character Recognition", arXiv, 2012.

[7] Neha J. Pithadia, Dr. Vishal D. Nimavat, "A Review on Feature Extraction Techniques for Optical Character Recognition”, IJIRCCE, 2015.

[8] http://hubpages.com/technology/Artificial-NeuralNetwork, Author: Ansonabey, Computer Engineer, Bangalore, India

[9] http://www.tribalengineering.com/technology/artificialneural-ictl.aspx. 